Revista Internacional de Apoyo a la Inclusión, Logopedia, Sociedad y Multiculturalidad.

Volumen 5, Número 2, Junio 2019, ISSN: 2387-0907.DOI: https://dx.doi.org/10.17561/riai.v5.n2.3

\title{
Buenas prácticas pedagógicas en la Institución Educativa Instituto Quimbaya (Quindío, Colombia): una mirada desde la formación del profesorado
}

(Good pedagogical practices in the Quimbaya Institute educational center (Quindío, Colombia): A look from the teaching training)

Eliana Alexandra Medina Moscoso

UNINI (Colombia)

yakaira18@yahoo.es

Fecha recepción: 21-03-2019

Páginas 25-39

Fecha aceptación: 20-05-2019

\section{Resumen.}

El presente artículo tiene por objeto identificar la influencia de la formación del profesorado en la aplicación de buenas prácticas pedagógicas en los niveles de preescolar, primaria y educación media del Instituto Quimbaya (Quindío, Colombia). La investigación es un resultado parcial de la tesis doctoral titulada "Buenas prácticas pedagógicas para una educación inclusiva en la institución educativa Instituto Quimbaya (Quindío, Colombia), cuyo objetivo general es analizar las prácticas pedagógicas de los docentes de la Institución Educativa Instituto Quimbaya, en pro de una educación inclusiva. Se tomó como base el método cuantitativo y cualitativo, con la aplicación el instrumento ACADI, en el aparte referido al Profesorado y sus indicadores B.1, B.2 y B.3. Los datos fueron analizados por medio del programa estadístico SPSS. Los resultados evidencian que en el Instituto Quimbaya no se desarrollan a cabalidad estrategias y prácticas de coordinación tanto entre docentes, los profesionales especialistas y el personal de apoyo, en la búsqueda de fortalecer las buenas prácticas pedagógicas.

Palabras clave: Educación inclusiva; buenas prácticas pedagógicas; formación del profesorado; prácticas de coordinación

\section{Abstract.}

The purpose of this article is to identify the influence of teacher training on the application of good pedagogical practices at the preschool, primary and middle school levels of the Quimbaya Institute (Quindío, Colombia). The research is a partial result of the doctoral thesis entitled "Good pedagogical practices for inclusive education in the Quimbaya Institute of Education (Quindío, Colombia), whose general objective is to analyze the pedagogical practices of the teachers of the Instituto Quimbaya Educational Institution, in pro of an inclusive education. The quantitative and qualitative method was taken as a basis, with the application of the ACADI instrument, in the section referring to the Teaching Staff and its indicators B.1, B.2 and B.3. The data were analyzed through the statistical program SPSS. The results show that the Quimbaya Institute does not fully develop co-ordination strategies and practices among teachers.

Keywords: inclusive education; good pedagogical practices; teacher training; coordination practices 


\section{1.-Introducción.}

Desde los años 80 investigadores de diversas partes del mundo e instituciones internacionales como la Unesco y la Organización de Estados Iberoamericanos para la Educación, la Ciencia y la Cultura, han promulgado el reconocimiento y la puesta en práctica de la educación inclusiva. Postulada como un valor humano, la inclusión requiere del reconocimiento de la diversidad étnica, social y cultural presente en los diversos territorios del mundo, por tanto, implica el derecho que tienen todos los niños y niñas, sin distinción de sus proveniencias económicas y culturales, ni sus diferentes capacidades, al buen trato y la igualdad de condiciones en la educación.

Si bien los Estados son los principales garantes del cumplimiento de este derecho, los distintos miembros que constituyen las sociedades son los llamados a hacerlo real y efectivo, y al ser los docentes, uno de los actores principales en la vida de los niños, es innegable su papel en el logro de la inclusividad. En este sentido, analizar las prácticas docentes que abordan y responden a la diversidad de las necesidades presentes en el aula de clase, 0 al contrario advertir las dificultades en este camino, es una labor relevante para hacer de la educación inclusiva una realidad.

El presente artículo contiene los resultados parciales de la tesis doctoral titulada "Buenas prácticas pedagógicas para una educación inclusiva en la institución educativa Instituto Quimbaya (Quindío, Colombia). Pretende hacer un aporte a la comprensión de los logros y obstáculos que la educación inclusiva tiene en la actualidad. Desde las miradas de las prácticas pedagógicas, advierte uno de los escenarios principales en el que la aceptación o la negación de la inclusividad tienen lugar: el aula de clase.

\section{2.-Referentes conceptuales.}

\section{1.-La educación inclusiva y la escuela inclusiva.}

La educación inclusiva es un bien al que todas las personas tienen derecho, por tanto, debe estar al alcance de todos. Promovida por instancias internacionales, la educación inclusiva ha sido definida como como una educación sostenida en un sistema de valores centrado en reconocer, apoyar y potenciar las cualidades y las necesidades los alumnos de la comunidad escolar de manera individual y colectiva. En este sentido la inclusión es también "un proceso que permite abordar y responder a la diversidad de las necesidades de todos los educandos a través de una mayor participación en el aprendizaje, las actividades culturales y comunitarias" (UNESCO, 2006, p. 14). Ella precisa de la identificación y la eliminación de barreras generadas por las creencias y actitudes presentes en contextos particulares, materializadas en culturas, políticas y prácticas escolares que generan exclusión, marginación 0 fracaso. Para el logro de tal propósito, la inclusión pone particular énfasis en aquellos grupos de alumnos que podrían estar en riesgo de marginalización, exclusión, 0 fracaso escolar. (Echeita \& Ainscow, 2011). 
La educación inclusiva requiere entonces del conocimiento de las características y particularidades de los grupos poblacionales diversos y vulnerables, que son atendidos en las instituciones educativas. Estas poblaciones se refieren a: étnicas (Afro descendientes, Rom y Raizales), con Necesidades Educativas Especiales (con discapacidad o con capacidades 0 talentos excepcionales), personas de talla baja (enanismo), jóvenes y adultos iletrados, afectadas por la violencia (en situación de desplazamiento, niños, niñas y jóvenes desvinculados de grupos armados al margen de la ley y adultos reinsertados), menores en riesgo social (niños, niñas y jóvenes trabajadores, adolescentes en conflicto con la ley, y niños y niñas en protección); habitantes de frontera y población rural dispersa.

Frente a este panorama social y cultural diverso, la inclusión implica "atender con calidad y equidad a las necesidades comunes y específicas que presentan los estudiantes". Para lograrlo se necesita la aplicación de herramientas que asuman la diversidad, afianzar concepciones éticas que "permitan considerar la inclusión como un asunto de derechos y de valores" e implementar estrategias de enseñanza flexibles e innovadoras que propendan por el logro de una educación personalizada, que desarrolle diversos estilos de aprendizajes y que asuma, de manera natural, que existen diversos niveles de desarrollo entre los estudiantes (Ministerio de Educación Nacional de Colombia, 2009, p. 9)

Referida a las personas en condición de discapacidad la inclusión se encamina a la contribución de dos propósitos:

Primero, aumentar el conocimiento que la persona tiene del mundo en que vive, al igual que su comprensión imaginativa tanto de las posibilidades de ese mundo como de sus propias responsabilidades en él; $y$, segundo, proporcionarle toda la independencia y autosuficiencia de que sea capaz, enseñándole con este fin lo necesario para que encuentre un trabajo y esté a disposición para controlar y dirigir su propia vida. (Warnock, 1987)

En consecuencia, como es planteado por Amstrong (1999) un sistema educativo inclusivo es aquel que "reconoce el derecho de todos los niños y jóvenes a compartir un entorno educativo común [... en el que sean] valorados por igual, con independencia de las diferencias percibidas en cuanto a capacidad, sexo, clase social, etnia o estilo de aprendizaje" (p. 76) Para tal alcance, la educación inclusiva debe generar estrategias que permitan atraer a los padres y a diversos actores sociales a las escuelas, de manera que se puedan ofertar alternativas tentadoras, desafíos que cautiven a los niños y niñas, que los alejen de toda posibilidad de desertar del sistema educativo. Esto requiere de la existencia voluntades y compromisos compartidos, donde la inversión y el esfuerzo sean de todos.

Según González Sánchez (2015), los siguientes son los aspectos que han ganado un consenso internacional respecto al concepto de inclusión: La asunción de determinados valores que deben presidir las acciones en pro de la inclusión; el reconocimiento de derechos; el incremento de la participación del alumnado en el 
currículo, en la cultura y en la comunidad y la prevención de cualquier forma de exclusión; la transformación de las culturas, la normativa y la prácticas educativas en pro del reconocimiento de la diversidad y de las necesidades particulares y contextuales de los estudiantes; el fortalecimiento de las escuelas con voluntad de "integrar" grupos vulnerables, y el desarrollo integral de los niños y niñas.

Para Flores, García y Romero (2017), la Escuela Inclusiva (EI) es un proceso que reestructura las políticas, culturas y prácticas; en ella se busca que el aprendizaje y la participación sean colectivas, es decir de todos los estudiantes, así como el involucramiento de toda la comunidad educativa con la intención de mejorar la escuela en beneficio de docentes y estudiantes. La implementación de la Escuela Inclusiva busca superar las barreras para el acceso y la participación; considera la diversidad como una riqueza para el proceso de enseñanza-aprendizaje. Sin embargo, su implementación exige un proceso situado, donde se parta del contexto institucional, de su historia, sus recursos y su capacidad de cambio, entre otras.

\section{2.-Las prácticas educativas y la formación del profesorado.}

Como lo plantea Ricoy (2018) la educación inclusiva no solo implica ampliar la cobertura al servicio educativo; ella va más allá de integración de las personas 0 grupos solo por su situación de vulnerabilidad en el sistema escolar, para exaltar la condición humana de cada participante. En palabras del autor,

Resignificar la educación en el enfoque de inclusión implica una mirada en dos sentidos. De un lado, de quien es el excluido y discriminado y de otro, ajustar las condiciones de contexto para hacer efectivo el derecho a la educación. La inclusión no es atribución exclusiva de las instituciones educativas, es un compromiso ético, una responsabilidad política y una condición necesaria para la viabilidad y la gobernabilidad en marcos de tolerancia. (p. 161)

En este sentido, las prácticas educativas son uno de los aspectos más importantes para lograr un verdadero proceso de inclusión. Ellas requieren que su planeación, basada en el currículo institucional, responda a cada una de las diferencias que se presentan en el aula. Por tanto, los maestros deben de ser co-desarrolladores del perfeccionamiento, tanto de su rol profesional como del currículo, para que los nuevos conceptos influyan en el perfil esperado de los estudiantes. Ellos deben ser promotores del compromiso que tienen todos los actores sociales, incluyendo los gobiernos con sus políticas, para establecer los mecanismos que propendan a la efectiva implementación de sistemas educativos inclusivos (Opertti, 2013). Para lograr estas condiciones, el proceso de formación de docentes debe proporcionar los elementos para generar cambios que permitan la construcción de estrategias de mediación que incluyan a todos los individuos que participan en el proceso educativo.

Para Duk y Murillo (2016) la inclusión es un concepto "dilemático", que tiene como eje central la diversidad humana vista desde una perspectiva sistémica y funcional, en la cual las decisiones y puesta en práctica, deben hacerse desde las políticas y 
prácticas educativas. La educación inclusiva tiene que ver con cada uno de los estudiantes, es decir con todos, quienes deben participar de una manera dinámica y permanente del proceso educativo. En este sentido, la educación inclusiva propende por remover barreras, transformar mecanismos excluyentes en incluyentes y potenciar el aprendizaje dentro del sistema (poiesis). Para los investigadores citados, en el desarrollo de tales propósitos surge un interrogante: ¿cómo lograr el difícil equilibrio ente lo común y lo diverso? Es decir, ¿cómo ofrecer una educación pertinente y real al contexto, que se ajuste a las diferencias socioculturales e individuales de los educandos y a la vez al pensamiento y a las dificultades cognitivas (sistema, escuela y aula), para que finalmente haya condiciones de igualdad y se dé una convivencia armónica, real y fructífera entre la diversidad, con pertenencia, y una verdadera participación social? En este interrogante confluye tres dilemas: el de la identificación o clasificación, el del agrupamiento y el dilema de la promoción o la repitencia.

La educación inclusiva es entonces un reto y en él, más aún, la labor del profesorado. Si bien en el mundo de hoy, existen grandes avances conceptuales, pedagógicos e institucionales que permiten no solo la integración, sino la inclusión de las diferencias, físicas, psicológicas, cognitivas, culturales y sociales a través del modelo de las escuelas inclusivas, aún falta que esto sea una realidad. Es decir, la inclusión no es aún un postulado que se desarrolle de manera completa en todas las instituciones escolares públicas y privadas de los diferentes países. Todavía son necesarios cambios importantes que van desde un currículo diversificado hasta posturas personales de los alumnos en su conjunto, de la sociedad, de los profesionales y de los gobiernos, para lo cual se requiere de compromisos gubernamentales, institucionales y pedagógicos.

Para Giné y Durán (2017), la educación inclusiva es "un proceso de formación, en un sentido amplio; un proceso de capacitación de los sistemas educativos, de los centros y del profesorado para atender la diversidad del alumnado" (p. 156). En este proceso de formación, es muy importante la que atañe al profesorado, "no como una tarea individual, sino como un proceso de desarrollo profesional y de mejora de los centros y los sistemas educativos" (Ricoy, 2018), ya que capacitar a los profesores para valorar y potenciar la diversidad implica un cambio en la cultura profesional de los docentes. Este cambio solo será efectivo si se realiza en un contexto asumido por los diversos actores del sistema educativo y orientado por valores inclusivos. La educación inclusiva precisa entonces de la toma de decisiones políticas y sociales encaminadas a "cambiar y modificar contenidos, enfoques, estructuras y estrategias, con un planteamiento común que incluya a todos los niños del grupo de edad correspondiente y con la convicción de que es responsabilidad del sistema general educar a todos los niños" (UNESCO, 2005, p. 13).

En consecuencia, siguiendo con Giné y Duran (2017), la formación del sistema educativo inclusivo debe orientarse hacia la preparación de profesionales que tengan la capacidad de reflexionar constantemente sobre el desarrollo de sus prácticas pedagógicas, llevadas a cabo a partir de dimensión ética interiorizada por ellos y por 
las demás personas que constituyen el centro educativo. Estos profesionales deben seguir sus prácticas educativas a partir de los siguientes criterios: Pedagogía de la complejidad, perspectivas constructivistas, desarrollo integrado, enseñanza adaptativa, adaptaciones curriculares, red de apoyos y colaboraciones, escuelas como centros de la mejora, diversidad como fuente de enriquecimiento y de estímulo para la innovación.

Por tanto, la inclusión tiene entre una de sus metas, "ayudar al profesorado a aceptar la responsabilidad de aprendizaje de todos los alumnos". Esto requiere de la identificación de las dificultades de los alumnos en el aprendizaje como oportunidades para mejorar la práctica educativa, es decir, ver en el obstáculo una oportunidad. En este sentido las prácticas pedagógicas encaminadas al logro de la educación inclusiva, deben surgir de las lecturas de los contextos particulares, en los que, tanto los elementos problemáticos como los favorables, son posibilitadores del aprendizaje (Giné y Duran, 2017).

La capacitación del profesorado, precisa de un cambio en la visión de su lugar dentro de la institución educativa en la que se contemple una visión transdisciplinar del currículo. Es necesaria la comprensión del profesor como eje fundamental en el proceso de inclusión, lo que implica que el oficio del profesor no solo sea una vocación, sino también una profesión que requiere transformaciones para trabajar con la diversidad y en apertura a la creación e implementación de prácticas innovadoras. Para ello es necesario que cada docente se apropie de los objetivos de la educación inclusiva, sea capaz de desafiar los estereotipos de la educación homogeneizadora, promueva el cambio social, y, desde el marco de la colaboración y la apertura a la diversidad, se proponga conseguir una mejor educación para todo el alumnado.

Por tanto, la educación inclusiva es un proceso que implica un cambio de paradigma educativo. Este cambio paradigmático debe dar como resultado una educación "basada en la heterogeneidad y no en la homogeneidad, considerando que cada alumno tiene una capacidad, interés, motivaciones y experiencia personal única, es decir, que "la diversidad está dentro de lo normal" (Souza Saburido, 2015, p.48). Para el logro de tales premisas educativas, las instituciones basadas en principios de inclusión requieren de profesores poseedores de conocimientos teórico-prácticos, pero además de planificaciones colectivas, estrategias y metodologías de enseñanza y de procesos de evaluación, presentes en el escenario institucional, que posibiliten al educador acompañar el desarrollo de cada alumno en su aula de clase (Souza Saburido, 2015).

\section{3.-Las buenas prácticas educativas.}

Como fue mencionado en apartados anteriores, desde una mirada holística, el sistema educativo debe responder por la realidad de todos los estudiantes, adaptándose a los contextos de cada uno. De esta manera, se espera que, sin excepción, los alumnos adquieran las competencias para participar en la vida social, 
política y económica; lo que requiere de una reforma que apoye y acoja la diversidad de todos los educandos y que permita un proceso efectivo y un cambio de metodologías constante, sin rutinas, en permanente movimiento y bajo la vigilancia continua de las prácticas existentes (Ainscow y Miles, 2008).

Al respecto, una de las dimensiones del proceso de inclusión, lo constituyen las Prácticas Inclusivas (PI) (Booth \& Ainscow, 2002). Estas comprenden las actividades propias del profesorado, que le permiten llegar al estudiante, transmitir el conocimiento, lograr el desarrollo integral de todos sus estudiantes y brindar una educación de calidad y pertinente a las necesidades y dificultades que se presenten. Las Prácticas Inclusivas son contextuales, es decir, se implementan en función de las necesidades del grupo, por tanto, las prácticas educativas pueden ser adecuadas 0 inadecuadas, pues lo que funciona para un grupo, puede no serlo en otro (Organización de Estados Iberoamericanos para la Educación, la Ciencia y la Cultura, 2009).

Para lograr su concreción, las Prácticas Inclusivas, deben cumplir requisitos como: diseñar diversos materiales teniendo en cuenta las características del alumnado, usar diferentes estrategias de enseñanza aprendizaje, poseer flexibilidad curricular, libertad para que el alumnado decida qué y cuándo evidenciar su aprendizaje, ofrecer variedad de actividades que motiven al alumnado, grados de complejidad para que por sí mismas favorezcan la diversificación y la organización óptima del tiempo. (Rubio, 2015, p. 40).

Así mismo, la Organización de Estados Iberoamericanos para la Educación, la Ciencia y la Cultura (OEI) (2009) define la importancia de realizar buenas prácticas inclusivas, entendidas como:

Una actuación "situada", que adquiere sentido y es viable a partir de una realidad concreta, de unos condicionantes estructurales que la hacen única e irrepetible. No hay buenas prácticas ideales sino que dependen del contexto en el que se desarrollan. [...] En consecuencia las "buenas prácticas" nos pueden "iluminar" el camino e ilustrar algunos tramos del recorrido; su finalidad es, pues, enriquecer nuestro horizonte a partir de las respuestas que otros han dado a problemas parecidos y no su imitación [...] Podemos concluir que constituye una "buena práctica", toda actuación que se oriente, a partir del compromiso del profesorado, el alumnado y las familias, a promover la presencia, la participación y el éxito de todo el alumnado, sobre todo de aquellos en mayor situación de vulnerabilidad. No es una cuestión de "todo o nada", sino fundamentalmente de "grado" y de progreso sostenido (proceso). (p. 5)

Para el Ministerio de Educación de Colombia (2009) las buenas prácticas pedagógicas son

Una serie de acciones que todo educador utiliza para dar respuesta a la diversidad: organizar el aula de manera que permita la participación y cooperación entre los 
estudiantes, dar alternativas de elección, ofrecer variedad de actividades y contextos de aprendizaje, utilizar materiales diversos, dar más tiempo a determinados estudiantes, graduar los niveles de exigencias y otras que son el resultado de la creatividad del docente. (p. 31)

Equiparando los conceptos de prácticas inclusivas, buenas prácticas inclusivas y buenas prácticas pedagógicas, en la presente investigación se ha adoptado el concepto de buenas prácticas pedagógicas, entendidas como todas aquellas prácticas presentes en el aula de clase que posibilitan la educación inclusiva. Es decir, todas aquellas prácticas que promueven y facilitan la integración de todos los niños sin importar sus diferencias físicas, cognitivas, culturales y económicas.

\section{3.-Metodología.}

Esta investigación de tipo mixto, se desarrolla a través de un estudio de caso, con el que, como sugiere Skate (2007), se pueden establecer diferencias entre dos modalidades: las mediaciones propuestas por el modelo pedagógico "Modificabilidad Estructural Cognitiva" (MEC) de Feuerstein, citado por Velarde (2014), y la forma como el docente desarrolla las prácticas pedagógicas. Las mediaciones son las variables para el análisis de resultados y el eje central de la investigación. Las prácticas pedagógicas es el otro punto de comparación, que será analizado a partir de la aplicación del Instrumento ACADI y que permitirá vislumbrar las 4 aristas en las que se enmarcan todos los procesos institucionales. Estas están contenidas en la guía 34 del Ministerio de Educación Nacional (MEN, 2008).

El ACADI"; es un instrumento de autoevaluación diseñado en España que reúne características psicométricas, valora la atención eficaz de la diversidad desde una mirada inclusiva; sus módulos permiten evaluar la institución en forma global o por ámbitos. Para su aplicación en la presente investigación el instrumento fue convalidado y ajustado por expertos de la Universidad del Quindío y de otros estamentos departamentales. El ACADI consta de una serie de preguntas agrupadas en 4 ámbitos, las cuales hacen parte de cada una de las gestiones del sistema educativo colombiano. Cada uno de estos ámbitos a su vez se divide en varios ítems, con 4 opciones de respuesta que reflejan la aplicación de estos en cualquier contexto educativo. Este instrumento se aplicó de manera individual, con previa explicación de lo contenido en cada instrumento, a 22 docentes desde los niveles de preescolar, básica y media. Para la sistematización de los datos y como base para el análisis, se empleó el método estadístico denominado IBM SPSS Statistics, versión 23 (IBM, 1989).

Los resultados concernientes a este artículo, a partir de la aplicación del instrumento ACADI fueron específicamente los correspondientes al Indicador B.3 concerniente exclusivamente al profesorado.

Las temáticas de esta categoría se enumeran a continuación: 


\section{Indicador: B.3.1.}

- Disponibilidad y práctica de estrategias de coordinación de profesores o existe una previsión de coordinación sistemática del profesorado para lo cual se prevén espacios y tiempos

\section{Indicador: B.3.2.}

- Se promueven actividades de formación permanente del profesorado 0 proyectos de innovación educativa vinculados a la mejora de la atención a la diversidad del alumnado, o se planifican, desarrollan y evalúan conjuntamente actividades de formación permanente (en el centro o fuera de él) así como de innovación educativa dirigidas, directa 0 indirectamente a mejorar la atención a la diversidad del alumnado.

- La dirección se implica en la promoción de las mismas, en la forma de incentivar en lo posible la participación de la mayoría del profesorado y en asegurar los medios y las medidas organizativas que faciliten su realización.

B.3.3. Profesorado de apoyo trabaja coordinadamente con tutores, y viceversa

- Existe coordinación entre profesorado especialista específico a las necesidades educativas especiales y tutores, y el resto de profesorado.

- El personal no docente (ATE, Fisio, ATS) participa en las reuniones de coordinación y en la toma de decisiones que en las mismas se llevan a cabo

\section{4.-Resultados.}

B.3.1. Estrategias y prácticas de coordinación entre docentes

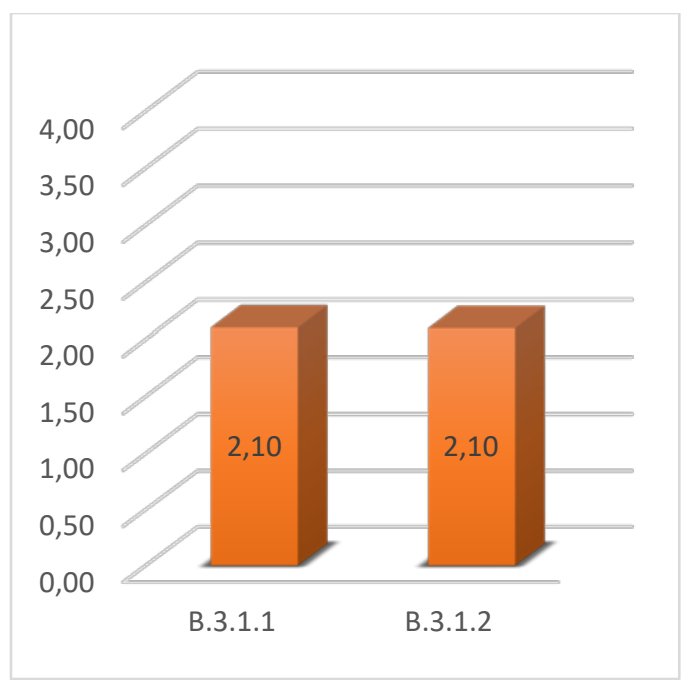

Figura 1. Media para el indicador B.3.1 
La media de los profesores tiende a responder y evidenciar la poca disponibilidad de estrategias coordinadas y prácticas en las que existen espacios y tiempos para un trabajo en equipo entre docentes y personal de apoyo. En las siguientes figuras se presentan los resultados para cada pregunta del indicador.

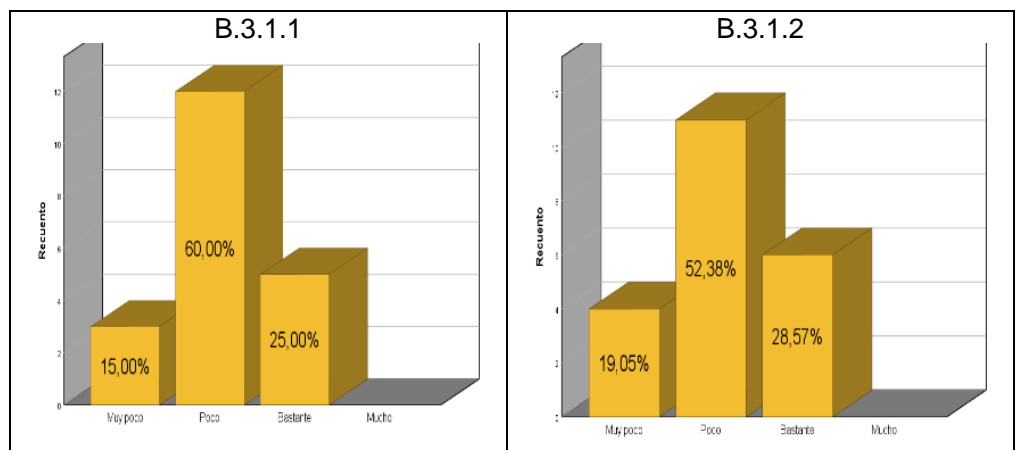

Figura 2. Resultados para el indicador B.3.1

El $75 \%$ de los docentes afirman que muy poco y poco se unifican estrategias educativas entre el profesorado para incidir en el grupo-clase. En el mismo sentido, respecto a la realización de reuniones periódicas de coordinación del equipo educativo con los profesores que atienden al mismo grupo de alumnos, pues el 71.43 $\%$ respondieron en el mismo rango (muy poco y poco).

Es evidente, entonces, que las buenas prácticas son únicamente la decisión y producción individual de los profesores, lo que dificulta el enriquecimiento de las actividades que favorecen la inclusión en términos generales en la institución.

B.3.2. Formación del profesorado para la realización de prácticas inclusivas.

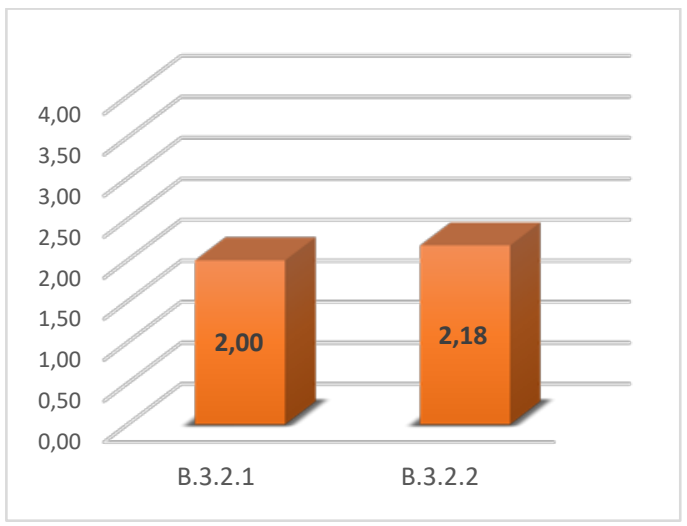

Figura 3. Media para indicador B.3.2 
Estos promedios indican valores muy bajos, que permiten inferir dificultades en su formación y propuestas institucionales para fortalecer los procesos inclusivos a partir de la capacitación docente.

En las respuestas, se evidencia que los docentes consideran que no existen actividades de formación o proyectos de innovación educativa que mejoren la atención a la diversidad del alumnado (81,8\%). Así mismo, que no tienen una buena percepción del trabajo que se adelanta en la institución para atender las dificultades de los estudiantes que requieren inclusión. El 68,19 \% de ellos expresa que muy poco y poco se promueven las actividades de formación permanente del profesorado 0 de personal de apoyo relacionada a la atención de la diversidad del alumnado.

Con estos resultados la posibilidad de diseñar estrategias innovadoras y aplicar mediaciones inclusivas se aleja más de una educación moderna basada en las particularidades de los estudiantes, pero en la vía de dar cabida a todos aquellos que llegan a la Institución Educativa.

B.3.3. Coordinación entre docentes, profesionales especialista y personal de apoyo

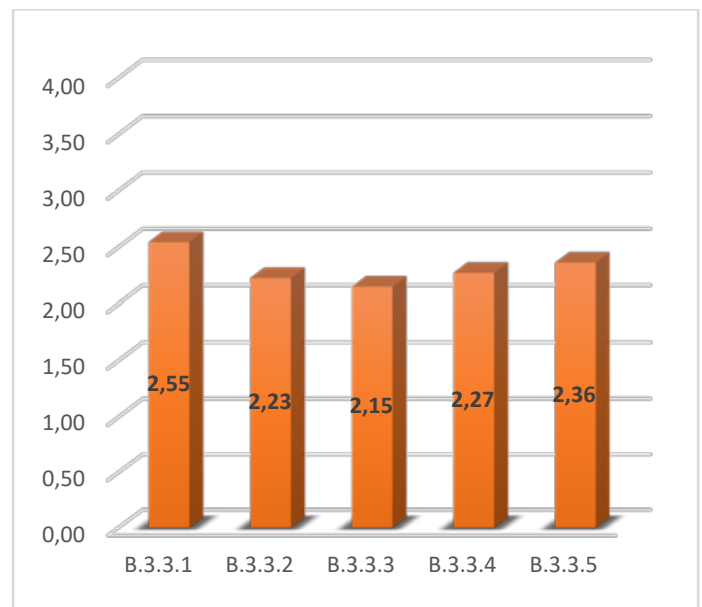

Figura 4. Media para las respuestas del indicador B.3.3

Los promedios de este indicador presentan un bajo desempeño de los docentes y demás profesionales en la construcción de planes conjuntos de intervención pedagógica. Para los docentes encuestados, aunque consideran que el personal de apoyo se preocupa por facilitar el aprendizaje y participación de los estudiantes, no se evidencia trabajo en equipo entre docentes y profesional de apoyo, ni comunicación asertiva para tratar a los estudiantes con necesidades de inclusión.

Aunque no existe capacitación por parte del Sistema educativo para favorecer los procesos de inclusión, los docentes en un $54,5 \%$ se preocupan (bastante y mucho) por facilitar el aprendizaje y la participación de los alumnos. 
La mayoría de los profesores (59\%) dan puntajes bajos (muy poco y poco) acerca del trabajo colaborativo en la búsqueda de mejoras pedagógicas para el éxito del alumnado, sin embargo, el resto de los maestros consideran que es bastante dicho trabajo.

EL 70\% de los docentes responden que son pocos o muy pocos los apoyos para responder a la diversidad, que deben ser considerados como una alternativa a los usuales apoyos individuales utilizados a los alumnos con necesidades especiales.

La respuesta sobre los acuerdos que se establecen entre los profesores de la institución y los servicios de apoyo externo (no se cuenta con ellos de modo habitual), en busca de mejorar los procesos de aprendizaje, es coherente con la pregunta anterior, ya que el $59,9 \%$ califican de muy poco o poco los acuerdos. El resto de las respuestas apuntan a mejor calificación, lo cual parece hacer referencia al apoyo interno con que cuentan los maestros en sus labores de aula.

Para los docentes hay divergencias entre la aplicación de las directrices claras sobre cómo organizarse para realizar el trabajo conjunto entre profesorado y personal de apoyo, ya que un $45,46 \%$ consideran que hay un trabajo de la Institución educativa bajo dichas directrices; mientras que el otro $54,5 \%$ de ellos considera que definitivamente no existe este trabajo.

B.3.4. Implicación colectiva en la concepción de la diversidad

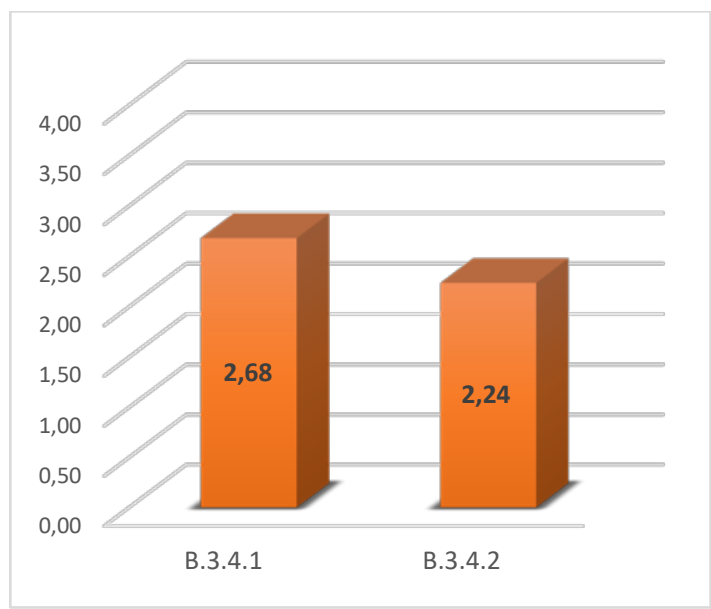

Figura 5. Media para las respuestas del indicador B.3.4

Los docentes consideran que se requieren acciones de apoyo para organizar los planes de mejora en la enseñanza y en el currículo, pero a la hora de organizarse no cuentan con dicho apoyo, en especial el del personal externo. El 59.09\% de los docentes afirman que sí existe un plan general de mejora de la enseñanza y del 
currículo para atender la diversidad de la Institución, sin embargo, el 66.67 de los profesores expresan que es muy poca la organización general de los apoyos para los profesionales de la Institución en los procesos de enseñanza aprendizaje, tanto interna como externa.

Denotan estas respuestas, que existe la voluntad para hacer acuerdos, pero solo se cuenta con un profesional de apoyo en todos los casos, pero no hay otros profesionales que aporten a la visión compartida ni apoyen las complejas tareas que deben enfrentar los profesores en el aula para lograr una real inclusión.

\section{5.-Discusión de resultados.}

Los resultados obtenidos evidencian que la Institución Educativa Instituto Quimbaya, no se desarrollan a cabalidad estrategias y prácticas de coordinación tanto entre los docentes, los profesionales especialistas y el personal de apoyo, en la búsqueda de fortalecer las buenas prácticas pedagógicas. Así mismo, es deficiente la formación del profesorado para la realización de prácticas inclusivas.

Como ha sido planteado por Amstrong (1999), la educación inclusiva precisa generar estrategias que permitan realizar acciones coordinadas entre los diversos actores educativos y sociales, lo que requiere de compromisos compartidos. La participación colectiva de los diferentes actores posibilita el mejoramiento de las condiciones pedagógicas educativas. En esta participación los maestros deben ser codesarrolladores de la educación inclusiva, tanto en su rol profesional como en la formulación y desarrollo del currículo institucional. La poca disponibilidad de realizar estrategias coordinadas entre los docentes y el personal de apoyo en el Instituto Quimbaya, advierte que este es un factor que dificulta el logro de las buenas prácticas educativas.

En la escuela inclusiva las acciones coordinadas se fortalecen con los procesos de formación de los docentes, ya que uno de los aspectos de dicha formación para el logro de la inclusión, es la adopción de elementos necesarios para generar cambios que permitan la construcción de estrategias de mediación entre los diversos actores del proceso educativo (Opertti, 2013). En este sentido los resultados de la investigación frente a los bajos niveles de capacitación docente en temas de inclusión por parte de la institución, se corresponden con la ausencia de coordinación.

La formación del profesorado implica, además, el desarrollo de valores que identifiquen y acepten la diversidad presente en los estudiantes, sea esta de carácter físico-cognitiva, étnica, económica, religiosa o de cualquier otro tipo. En la escuela inclusiva, la preparación del profesorado y el personal de apoyo debe enfocarse desde una dimensión ética que potencie la capacidad de reflexión constante frente a las prácticas pedagógicas. Estas deben emanar del reconocimiento del contexto educativo particular y del reconocimiento de elementos problemáticos como posibilitadores del aprendizaje (Giné y Duran, 2017). En el Instituyo Quimbaya el alto porcentaje de docentes (81.1\%) que consideran que no existen actividades de 
formación o proyectos de innovación educativa tanto para ellos como para el personal de apoyo, es un indicador de una falencia institucional en el logro de la educación inclusiva. Así mismo, es una falencia institucional la escasa presencia de apoyos para responder a la diversidad. Para un porcentaje alto de docentes, estos apoyos no se cuentan de un modo habitual, lo que se relaciona con la débil o nula existencia de un trabajo conjunto entre el profesorado y el personal de apoyo. Sin embargo, frente a esta problemática, los docentes manifiestan que ellos sí se preocupan por implementar buenas prácticas pedagógicas que facilitan el aprendizaje y la participación de todo el alumnado.

\section{6.-Conclusiones.}

La aplicación de buenas prácticas pedagógicas por parte de la comunidad educativa, requiere de la coordinación de estrategias y acciones conjuntas, de igual manera, precisas de gestiones institucionales para la existencia de una formación permanente del profesorado. El bajo logro de estos aspectos en el Instituto Quimbaya, desde las consideraciones del profesorado, advierte algunos de los elementos institucionales a mejorar para el logro total de la educación inclusiva en pro de su consolidación como una escuela inclusiva.

Esto se contrasta con los esfuerzos reconocidos por los mismos maestros de sus acciones particulares en pro de la implementación de buenas prácticas pedagógicas. Sin embargo, la educación inclusiva requiere del compromiso y esfuerzo de todos los actores educativos. No es posible construir una escuela inclusiva sin el involucramiento ético y práctico de la totalidad actores educativos y sociales.

Al ser este artículo un abordaje parcial de la tesis doctoral mencionada, estos resultados serán contrastados con las consideraciones de los demás actores institucionales. Sin embargo, estos advierten, de entrada, una mirada del escenario de aplicación de las buenas prácticas pedagógicas desde un actor fundamental de la educación inclusiva: el docente.

\section{7.-Referencias.}

Ainscow, M., y Miles, S. (2008). Por una educación para todos que sea inclusiva. Perspectivas, 38 (1), 15-44.

Armstrong, F. (1999). Inclusion, curriculum and the struglle for space in school. International Journal of Inclusive Education, 3(1), 75- 87.

Booth, T. \& Ainscow. M. (2002). Index for inclusion (2nd ED). Developing leaning and participation in schools (2aed). Manchester: CSIE [trad. Guía para la evaluación y mejora de la educación inclusiva. Madrid: Consorcio Universitario para la Educación Inclusiva.

Duk, C., y Murillo, F. J. (2016). La Inclusión como Dilema. Revista latinoamericana de educación inclusiva, 10 (1), 11-14. 
Echeita, G., y Ainscow, M. (2011). La educación inclusiva como derecho. Marco de referencia y pautas de acción para el desarrollo de una revolución pendiente. Tejuelo: Revista de Didáctica de la Lengua y la Literatura 12 (2011): 26-46

Flores Barrera, V. J., García Cedillo, I., Romero Contreras, S. (2017) Prácticas inclusivas en la formación docente en México Liberabit. Revista de Psicología Universidad de San Martín de Porres Lima, 23 (1), 39-56

Giné C. y Durán Gisbert, D. (2017). La formación del profesorado para la educación inclusiva. Recuperado de: <http://www.repositoriocdpd.net:8080/ bitstream/handle/123456789/1913/Art_DuranGisbertD_Formaciondelprofesor ado.pdf?sequence $=1>$.

González Sánchez, David. (2015) Análisis descriptivo y orientaciones prácticas hacia la inclusión escolar. Un estudio de centros educativos de Castilla y León. Tesis de grado. Programa de doctorado Psicología y Ciencias de la Educación. Universidad de León

Ministerio de Educación Nacional de Colombia. (2009). Guía 11. Educación Inclusiva con calidad. "Construyendo capacidad institucional para la atención a la diversidad". Bogotá

Opertti, R. (2013). La educación inclusiva, perspectiva internacional y retos de futuro. Lima: Proyecto Libro Digital.

Organización de Estados Iberoamericanos para la Educación, la Ciencia y la Cultura (OEI) (2009). Guía para la reflexión y la valoración de prácticas inclusivas.

Ricoy, Andrés. (2018). Formación docente y su relación con los contextos inclusivos. Revista Internacional de Apoyo a la Inclusión, Logopedia, Sociedad y Multiculturalidad. Volumen 4, Número 2, Abril 2018, ISSN: 2387-0907, Dep. Legal: J-67-2015

Rubio, S. (2015). La participación colaborativa de las familias a través de las prácticas inclusivas (Tesis de maestría). Universidad de Valladolid. Segovia, España.Recuperado de https://uvadoc.uva.es/bitstream/10324/13477/1/TFMB.96.pdf

UNESCO. (2006). Orientaciones para la Inclusión: Asegurar el Acceso a la Educación para Todos. París

Warnock, M. (1987). Encuentro sobre Necesidades de Educación Especial. Revista de Educación, Número Extraordinario, pp. 45-73. 